\title{
PATHOLOGICAL REMARKS
}

\author{
ON THE KIND OF \\ PALPEBRAL TUMOUR \\ USUALLY CALIED, IN ENGLAND, TARSAL TUMOUR. \\ BY \\ H. HAYNES WALTON, Esq., F.R.C.S., \\ SURGeON TO THE CENTRAL LONDON OPHTHALMIC hOSPITAL; \\ ASSISTANT-SURGEON TO ST. MARY'S HOSPITAL. \\ Received Nov. 28th, 1853.-Read Jan. 24th, 1854.
}

Tre subject of tumours of the ocular appendages is obscurely treated of by writers, more, I believe indeed, than any in the whole range of ophthalmological literature. The several stages of the same affection are described as different diseases, and the same diseases are dissimilarly delineated. A Greek and a Latin word bearing the same signification,-Chalazion and Grando, are applied to different morbid states. The disease I now propose to treat of is involved in similar perplexity; for I find it spoken of as "fibrinous tumour," "tarsal tumour not encysted," "albuminous tumour," and by other terms equally erroneous. There is also disagreement concerning its connection, whether moveable or not, and even as to consistence, whether hard or soft. I propose, therefore, in order to be understood respecting the tumour I mean, to describe the most palpable objective characteristics, before I point out what appears to be its pathological condition, and which has not, so far as I am aware, ever been demonstrated.

Commencing, then, with the external characters, I would speak of it as a hard, spherical, well-defined tumour, in size 
varying from that of a grain of small shot to that of a pea, and limited to a position on the eyelid corresponding to the space bounded by the cilia bulbs, and the upper margin of the tarsus; that is, corresponding to the position of the meibomian glands, not growing at the edge, and immoveable. Inadherent to the skin, which may or may not be traversed by enlarged blood-vessels, being usually solitary, and for the most part growing on the upper eyelid, yet acquiring the largest dimensions on the under, where the skin is generally in the natural state; not unfrequently giving, on the internal surface of the eyelid, indication of its existence by a spot of preternatural redness, and at a later period discoloration, or even a small fungous growth.

In proceeding now to its pathology, I must at starting acknowledge the assistance that $I$ have here received from Dr. Druitt. A very marked example of the tumour on the upper eyelid in a male, æt. 53, having come under my care in the summer of this year, $I$ turned the skin aside and removed it, together with the corresponding portion of the tarsus, and sent it to him for investigation, as he was at the time working on the subject of tumours. It may be well, before I subjoin his valuable report, to state, that the wound was brought together with sutures, and healed quickly, not only without disfiguration, but without leaving a scar.

"The tumour," he says, "was oval, $\frac{17}{48}$ ths of an inch in its length, and $\frac{13}{48}$ ths in its short diameter; having on the one side the entire thickness of the tarsal cartilage (so called) with the conjunctiva, on which a few meibomian follicles projected in the form of yellow granules; and on the other, some fibres of the orbicularis. It was evident that the growth was most intimately adherent to the (so called) tarsal cartilage. On bisecting it by a clean incision, some viscid, puriform fluid escaped. On closer examination, the centre appeared to be constituted by a very clear transparent membranous cyst, almost $\frac{1}{12}$ th of an inch in diameter, containing the aforesaid puriform fluid, and in the very midst, a small perfectly smooth circular pellet of sebaceous matter. Around this cyst was a soft pinkish material, and this again was con- 
tained within a tough fibrous capsule, continuous with the fibrous envelope of the (so called) tarsal cartilage." He continues: "On making further sections, and examining them microscopically I perceived-1st, The conjunctival surface covered with epithelium, several branches of meibomian follicles projecting like villi, these follicles being filled with solid or liquid sebaceous matter, and constituting the yellow granular bodies visible to the naked eye. $2 \mathrm{~d}$, I noticed the proper fibrous tissue, commonly called cartilage, of the lid, the fibres for the most part running parallel with the conjunctival surface; it was abundantly permeated by vessels, and contained in spherical loculi, bunches of meibomian follicles. $3 \mathrm{~d}$, These follicles, except that some projected, as aforesaid, like bunches of currants, on the conjunctival surface were mostly contained in spherical fibrous loculi within the fibrous membrane. Some contained soft, others hard matter. 4th, The tumour itself, consisting externally of a dense, fibrous cyst, continuous with the fibrous tissue of the lid; within this a layer of fibro-plastic matter, soft, pink, abundantly supplied with vessels from the fibrous cyst, composed of fibro-plastic cells, with a very little intercellular fibrillary matter; within this, the thin pellucid cyst above mentioned, containing a puriform fluid, made up of pus globules, epithelium cells loaded with oil, and in the centre a perfectly round pellet of sebaceous matter." In conclusion, he suggests the following to be the order of development: " 1 st, The formation, with a meibomian follicle, of a pellet of hard sebaceous matter. 2d, The secretion of a more copious epithelium and fluid matter around. 3d, The addition of fibro-plastic matter around the obstructed gland follicle, distending the loculus of fibrous membrane into a cyst."

Through the liberality of the Museum Committee of the Royal College of Surgeons, I have been allowed, in conjunction with Mr. Quekett, to examine two tumours of this class belonging to the College Museum, whereby the accuracy of Dr. Druitt's statement is verified, and other facts have been elicited. It was quite impossible, while these specimens were in the bottles, to understand them, and the references 
to them in the Catalogue, is not descriptive. I mention this, because I have, elsewhere, rather misrepresented them. In the one, on the outside of the tarsus from which the skin and the orbicularis muscle are removed, are two growths, one very small, too minute, perhaps, to have been recognised in life, and overlapped by the greater, many sizes larger; which, although firmly incorporated with the tarsus, holds its union by a small base. On the inside of the tarsus, the site of each is plainly marked by yellowish deposits in the course of the meibomian glands. Both were cut across, and found to contain epithelium scales and sebaceous matter. I beg to direct attention to the circumstance that the lesser, which is just enough developed to admit of a distinctive character, is equally well marked within the lid as the larger.

The other specimen afforded less definite information, yet it was peculiar and also instructive. The tumour occupied the entire upper eye-lid, from which the tarsus had nearly disappeared. It consisted, on the external or upper surface, of a dense fibrous sac: on the inside, that is within the lid, of conjunctiva. The interior, which was irregular and cryptlike, was, as in the other tumours, filled with epithelium scales and sebaceous matter. It would seem here, as if the entire meibomian apparatus had been simultaneously diseased.

I submit to the Society whether, if they consider the pathology of the disease to be proved, it would not be judicious to institute the term meibomian tumour, and to adopt a name alike simple, correct, and significant, an advantage not often to be met with in the nomenclature of ophthalmic literature, which is for the most part abominable and barbarous.

I have often heard it advanced in argument against the tumour originating in the meibomian glands, that it is always on the outside of the tarsus. This erroneous statement is advanced on the false supposition of the situation of these glands. It has long been pointed out that they are imbedded in the tarsus; however, anatomical works state differently, 
and describe them as seated between the conjunctiva and it. The entire glands are within the tarsus, their ducts even traverse it, and open on its free margin. The relative anatomy of the parts may to a certain extent be seen with the naked eye, for if the tarsus be dissected out, the glands will be equally visible on either side.

It appears to me that the determination of the tumour outwards, depends on the same law that causes the elimination outwards of foreign substances from the body, and includes in its operation the directing of morbid growths to the surface. That there is occasionally an exception to the law, the tumour taking an inward direction, and appearing on the side of the eye-lid, all surgeons engaged in ophthalmic practice are aware. It then appears in an arrested state, which may be thus explained. The discoloured spot on the interior of the eye-lid, above spoken of as giving internal indication of existence of the tumour, is produced by absorption of the tarsus, solely, I believe, in consequence of the pressure produced by the tumour on the eye-ball, and hence the upper eye-lid, as it is more in contact with the eye-ball, usually exhibits this change earlier and in a more marked degree. This may not proceed beyond a very limited extent, but occasionally much of the tarsus is removed when the determination inward would seem inevitable. With the removal of the tarsus, the chief and densest covering is lost, and perhaps as a consequence, there is no deposit of the fibroplastic material. The conjunctiva, then the only envelope, if not interfered with by art, is apt, like the tarsus, to suffer from absorption and ulceration, allowing the exposure of the distended follicle, which in turn is similarly affected, and discharges its morbid contents, or throws out a fungus growth.

It is well known that meibomian tumours may contain dissimilar substances, or a mixture of them; we meet with glairy, sebaceous, creamy, or purulent deposits ; and that the amount does not always bear an uniform relation to the size of the tumour, there being sometimes scarcely any fluid in a very large one, the sac being filled with a solid material. 
On this point I venture to suggest, that some of these characters depend on the changes effected in the fibro-plastic material that is deposited. For instance, with the onward development of the plastic material, white, or yellow fibrous tissue is produced, as in the tumour commonly called polypus ; hence the more solid tumour. Or, if it degenerate and undergo retrograde metamorphosis, the cells are converted into pus, or pyoid cells, and the inter-cellular tissue into a creamy fluid.

P.s.-I desire to say, that since I have adopted the pathological views above stated, I have, whenever surgical measures are required, ceased to employ in general the usual method of attacking the meibomian tumour from the interior of the eye-lid; but for the most part, that is, when the tumour takes an outward direction, I divide it on the outside, squeeze out the contents, and when it can be accomplished, pull away the cyst with a pair of forceps, or if necessary, remove it by dissection. I am enabled to assert, that this process is far superior to the other, insomuch as it is instantaneously effectual. It is not necessary for me to point out the tediousness and uncertainty of the older method. I must add, that there need be no fear respecting the formation of a scar on the eye-lid; for if the incision be made horizontally, and the edges be brought together by a strip of plaster, no trace of the operation is left. 